\title{
Role of diffusion MRI in diagnosis of mediastinal lymphoma: initial assessment and response to therapy
}

\author{
Youssriah Yahia Sabri ${ }^{1}$, Nourhan Mohamed Ewis ${ }^{1 *}$, Hussam Eldin Hamdy Zawam² and Mostafa Ahmed Khairy ${ }^{1}$
}

\begin{abstract}
Background: Malignant lymphoma accounts for nearly $20 \%$ of all mediastinal neoplasms in adults and $50 \%$ in children. Hodgkin's disease is the most common primary mediastinal lymphoma. In non-Hodgkin's lymphoma, the two most common forms of primary mediastinal lymphoma are lymphoblastic lymphoma and diffuse large B-cell lymphoma. The aim of this study is to implement diffusion MRI in the algorithm of diagnosis of mediastinal lymphoma, differentiating Hodgkin's from non-Hodgkin's lymphoma and assessment of post therapeutic response.
\end{abstract}

Results: Using Diffusion weighted magnetic resonance imaging DWI-MRI, there were statistic significant difference between ADC values in lymph nodes and mediastinal masses in Hodgkin and non-Hodgkin lymphomas. ADC range in non-treated Hodgkin lymphoma cases was 0.774 to 1.4 , while ADC range in in non-treated non-Hodgkin lymphoma was 0.476 to 0.668 . In this study, there was statistically significant difference of ADC values in lymphoma cases presented by mediastinal masses with and without chemotherapy.

Conclusions: Diffusion weighted magnetic resonance imaging DWI-MRI is a promising functional technique in diagnosis of Hodgkin's and non-Hodgkin's lymphoma and assessment of response to treatment with no need for special preparation, contrast injection or radiation exposure.

Keywords: Mediastinum, Malignant, Lymphoma, Hodgkin, Non-Hodgkin, Diffusion, MRI

\section{Background}

Malignant lymphoma accounts for nearly $20 \%$ of all mediastinal neoplasms in adults and $50 \%$ in children. Hodgkin's disease is the most common primary mediastinal lymphoma. In non-Hodgkin's lymphoma, the two most common forms of primary mediastinal lymphoma are lymphoblastic lymphoma and diffuse large B-cell lymphoma [1].

Mediastinal lymph nodes are divided into 7 zones and 14 stations as defined by the International Association for the Study of Lung Cancer (IASLC) [2, 3]. However, it can be used in lymph node classification in lymphoma [4].

\footnotetext{
*Correspondence: nourhan.ewis@kasralainy.edu.eg

${ }^{1}$ Department of Diagnostic and Interventional Radiology, Kasr Al Ainy,

Faculty of Medicine, Cairo University, Giza City, Egypt

Full list of author information is available at the end of the article
}

New MRI techniques, such as MRI diffusion weighted imaging (DWI), may be an alternative to CT and FDG$\mathrm{PET} / \mathrm{CT}$ in diagnostic imaging protocols for malignant lymphoma [5].

Diffusion refers to the random Brownian motion of water molecules [6]. T2-weighted images are converted to diffusion weighted images by applying diffusion gradient pulses before and after the $180^{\circ}$ radiofrequency pulse. The $b$ value (in seconds per square millimeter) depends on and increases with amplitude, duration of application, and separation of diffusion gradient [7]. Calculation of ADC from DW-MRI is measured by performing DWMRI with at least two diffusion weightings, or $b$ values. The differential signal attenuation at different $b$ values can be used to calculate the ADC [8].

In Hodgkin lymphoma the most commonly affected nodes include the prevascular, paratracheal and to less 
extent the hilar and subcarinal lymph nodes, while in non-Hodgkin lymphoma the most common lymph nodes involved are prevascular and paratracheal followed by the subcarinal, hilar, internal mammary, pericardial and posterior mediastinal nodes [9].

In Hodgkin lymphoma, thymic lymphoma and the involved nodes usually reveal homogeneous low signal intensity on T1-weighted images [10]. On T2-weighted MR images, they usually demonstrate homogeneous high signal intensity [11]. The mean ADC value of Hodgkin lymphomas is $\left(1.07 \pm 0.11 \times 10^{-3} \mathrm{~mm}^{2} / \mathrm{s}\right)[12]$.

While in non-Hodgkin lymphoma, the tumors appear as a large anterior mediastinal mass in nearly all patients. Solid portions of lymphoma are hypo intense in T1-weighted images but iso intense in T2-weighted images [13]. The mean ADC value for non-Hodgkin lymphoma is $\left(0.97 \pm 0.24 \times 10^{-3} \mathrm{~mm}^{2} / \mathrm{s}\right)[12]$.

In mediastinal lymphomas, a residual mass is common after treatment, especially in cases with a bulky initial mass and MRI provides important information in differentiation viable tumors from residual benign masses [14].

Mediastinal masses span a wide histopathological and radiological spectrum. While relatively specific imaging clues exist for certain lesions such as thyroid masses and cysts, many solid malignant and benign lesions appear remarkably similar on computed tomography $(\mathrm{CT})$ and magnetic resonance imaging (MRI) [15].

The major differential diagnoses of anterior mediastinal solid tumours in adults include thymic epithelial tumor, germ cell tumor, lymphoma and intra-thoracic goiter [16].

Differentiating lymphoma from sarcoidosis in mediastinal and hilar lymphadenopathy can be done by detecting the ADC value of the enlarged lymph nodes, the ADC value in the lymphoma was detected to be lower than that in the sarcoidosis group [17].
The aim of this study is to implement diffusion MRI in the algorithm of diagnosis of mediastinal lymphoma and assessment of post therapeutic response.

\section{Methods}

This cross sectional study involved 32 patients diagnosed as lymphoma; 18 males and 14 females, age range 18-69 years (average of 34.375 years). The complaints varied between dyspnea, chest pain, purities, fever and loss of weight.

Cases were referred from clinical oncology department to radiology department for MRI chest with diffusion in the period from December 2018 to March 2020.

\section{Inclusion criteria}

Patient diagnosed to have mediastinal lymphoma by biopsy and histopathological analysis.

\section{Exclusion criteria}

Contraindication to MRI e.g. Patients with pace maker, cochlear implants, cerebral aneurysm clips, ocular metallic foreign body, bullets or gunshots near great vessels or vital organs.

\section{MRI technique}

The MR scanning sequences were T1W1, T2WI, T2 STIR and DWI including quantitative DWI analysis (ADC measurement). Respiratory gating has been used in all patients. MR imaging parameters are listed in Table 1.

\section{Assessment of cases}

Three cardiothoracic radiologists with 5 (reader 1), 10 (reader 2) and more than 25 (reader 3 ) years of experience, respectively, interpret the images. Disagreements among readers regarding the images were resolved by consensus after a discussion between both readers and

Table 1 MR imaging parameters

\begin{tabular}{|c|c|c|c|c|c|c|}
\hline & TR/TE (ms) & $\begin{array}{l}\text { Direction of frequency } \\
\text { encoding }\end{array}$ & $\begin{array}{l}\text { Section thickness } \\
(\mathrm{mm})\end{array}$ & $\begin{array}{l}\text { Inter-slice gap } \\
(\mathrm{mm})\end{array}$ & FOV (mm) & Matrix \\
\hline Axial T1-WI (TSE) & $10 / 5$ & AP & 8 & .5 & $420 \times 325 \times 306$ & $288 \times 224$ \\
\hline Coronal T1-WI (TSE) & $10 / 5$ & $R / L$ & 8 & .5 & $425 \times 425 \times 208$ & $288 \times 224$ \\
\hline Axial T2-WI (TSE) & $644 / 80$ & AP & 8 & 1.5 & $420 \times 325 \times 306$ & $288 \times 224$ \\
\hline Coronal T2-WI (TSE) & $644 / 80$ & $R / L$ & 8 & 1.5 & $425 \times 425 \times 208$ & $288 \times 224$ \\
\hline Sagittal T2-WI (TSE) & $644 / 80$ & AP & 8.8 & 1.5 & $400 \times 299 \times 284$ & $288 \times 224$ \\
\hline STIR-WI & $1.6 / 20$ & $A P$ & 10 & 1 & $450 \times 333 \times 334$ & $288 \times 224$ \\
\hline $\begin{array}{l}\text { DWI (SS-SE-EPI-with fat } \\
\text { suppression) }\end{array}$ & \multicolumn{6}{|c|}{$\begin{array}{l}\text { DWI were acquired in a transverse plane, using three } b \text { values: low }\left(0-50 \mathrm{~s} / \mathrm{mm}^{2}\right) \text {, intermediate }\left(500-800 \mathrm{~s} / \mathrm{mm}^{2}\right) \text { and high } \\
b \text { value }\left(1000 \mathrm{~s} / \mathrm{mm}^{2}\right)\end{array}$} \\
\hline & $1407 / 66.5$ & AP & 5.9 & 1.5 & $420 \times 324 \times 306$ & $140 \times 107$ \\
\hline ADC map & \multicolumn{6}{|c|}{$\begin{array}{l}\text { ADC maps were calculated by the MR system via linear regression analysis of the natural log of signal intensity using all } \\
\text { three } b \text { values }\end{array}$} \\
\hline
\end{tabular}


a third chest radiologist with more than 25 years of experience (consensus decision).

\section{A. Patient grouping}

- Cases were divided into two groups according to receiving chemotherapy or not. Non treated group include 18 cases while treated group include 14 cases.

\section{B. Image assessment Qualitative analysis:}

- MR images qualitatively analyzed by visual assessment of the different pulse sequences and considering the suspicious areas of interest. Areas of restricted diffusion are bright on DWI using high $B$ value and dark on ADC map indicating low ADC value. The visual assessment including the location of the affected group of lymph nodes and associated MR imaging findings e.g. pleural effusion.

\section{Quantitative analysis:}

- ADCs were calculated from the ADC maps, which were constructed from $b=0$ and $b=1000 \mathrm{~s} / \mathrm{mm}$ values. A region of interest (ROI) was drawn within the lesion on the ADC map. ROI positioned within areas of the most diffusion restriction on visual judgment, trying to avoid as much as possible obviously cystic or necrotic areas. Three ROIs were placed and the average of three measurement was recorded.

\section{Statistical methods and data analysis}

- Data analysis packages used were SPSS packages version 21 and Medcalc version 19.2. Qualitative data were presented by number and percentage while quantitative data were presented by average and range, Bar chart and box plot were used to display data accordingly. Statistical tests were done for qualitative data: Chi square test with Fischer exact for qualitative data and Mann-Whitney test for quantitative non-normally distributed data. Level of significance was considered if $p$ equal to or below 0.05 ROC curve was used to detect validation of ADC of Hodgkin versus non-Hodgkin lymphomas. Youden index test was to detect cut odd point of ADC value, specificity, sensitivity, positive predictive value and negative predictive value as well as negative like hood ratio.

\section{Results}

This study involved 32 patients; 18 males and 14 females, age range 18-69 years (average of 34.375 years). All the patients diagnosed as lymphoma by histopathology. Cases were referred from clinical oncology department to radiology department for MRI chest with diffusion in the period from December 2018 to March 2020.

\section{Non-treated group}

- Cases were grouped according to their histopathological diagnosis into Hodgkin and non-Hodgkin lymphoma).

- Twelve cases diagnosed as Hodgkin while six cases diagnosed as non-Hodgkin lymphoma.

\section{A. Hodgkin lymphoma}

Six cases presented by discrete and partially amalgamated lymph nodes; while six cases presented by anterior mediastinal mass. There were affection of different groups of lymph nodes (Table 2).

ADC range in non-treated Hodgkin lymphoma cases presented with lymph node presentation was 0.83 to 1.4 with ADC average 1.1072, while ADC range in cases presented with masses was 0.774 to 1.1 with average 0.939 (Fig. 1).

\section{B. Non-Hodgkin lymphoma}

One case presented by lymph nodes affection including internal mammary and supra-clavicular lymph nodes; while five cases presented by mediastinal mass (Table 3).

ADC range in non-treated non-Hodgkin lymphoma cases presented with lymph node presentation was 0.476 to 0.548 with ADC average 0.512 , while ADC range in cases presented with masses was 0.507 to 0.668 with average 0.5946 (Fig. 2).

Table 2 Summary of lymph nodes distribution in non-treated Hodgkin lymphoma

\begin{tabular}{lll}
\hline Group of lymph node & Number of cases & Percentage \\
\hline Paratracheal & 5 & 19 \\
Prevascular & 4 & 15 \\
Paraaortic & 2 & 8 \\
Subaortic & 3 & 11.5 \\
Hilar & 3 & 11.5 \\
Sub carinal & 2 & 8 \\
Para esophageal & 4 & 15 \\
Cardiophrenic & 1 & 4 \\
Internal mammary & 1 & 4 \\
Supra clavicular & 1 & 4 \\
\hline
\end{tabular}



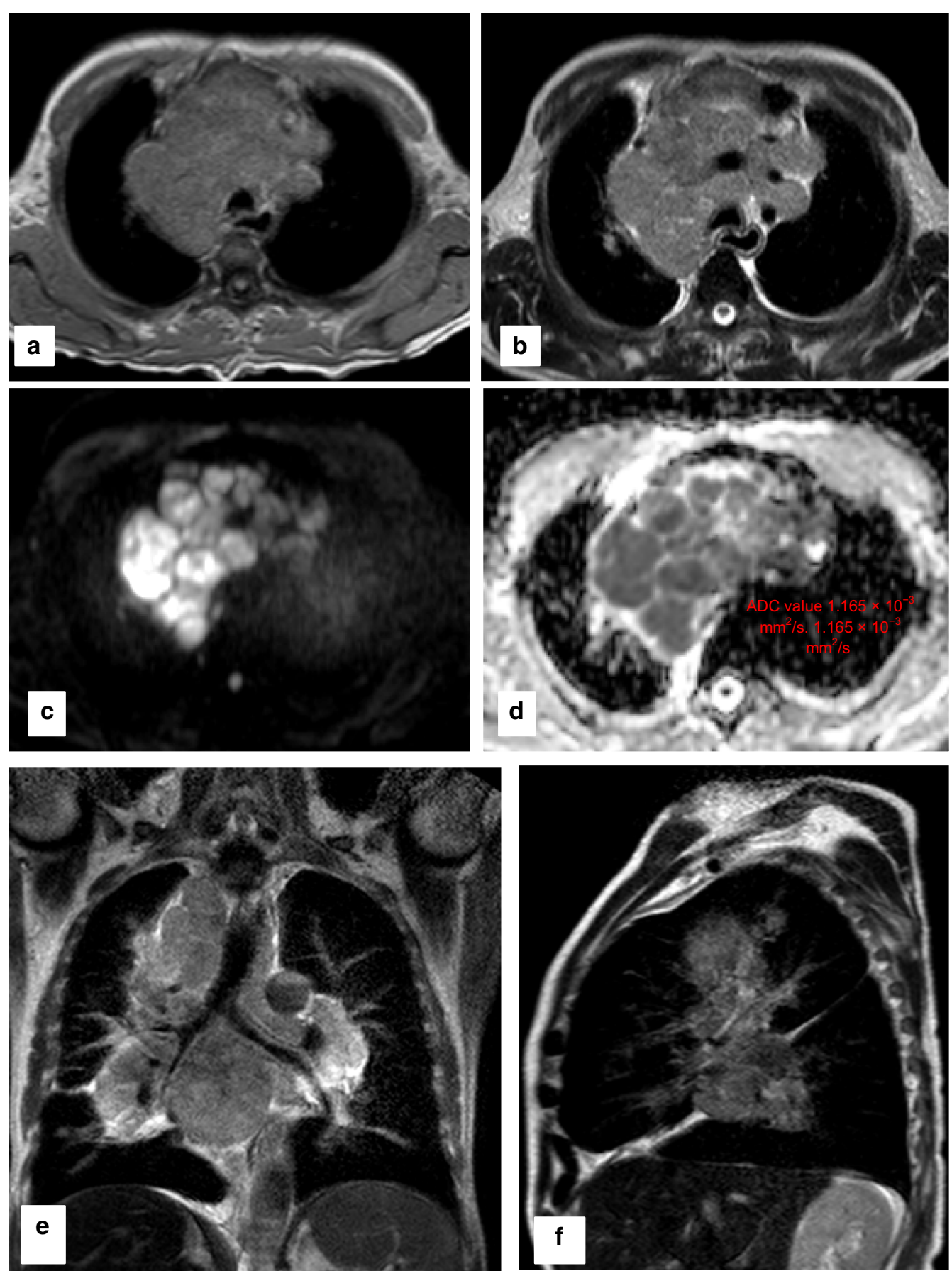

Fig. 1 A 65 year old male patient diagnosed as Hodgkin lymphoma. MRI was done before onset of therapy. a-d: a Axial T1 WI, b axial T2 WI, c axial DWI and $\mathbf{d}$ ADC map show amalgamated prevascular and paratracheal lymph nodes. They are isointense on T1 and T2 WI, hyperintense on DWI and hypointesne on ADC map denoting restricted diffusion with ADC mean value $1.165 \times 10^{-3} \mathrm{~mm}^{2} / \mathrm{s}$. e Coronal T2 WI and f Sagittal T2 WI showing multiple amalgamated mediastinal lymph nodes and right fissural pleural effusion 
Table 3 Summary of masses distribution in non-treated nonHodgkin lymphoma

\begin{tabular}{lll}
\hline Mass & Number of cases & Percentage \\
\hline Anterior mediastinal & 3 & 60 \\
Anterior and posterior & 1 & 20 \\
Anterior middle and posterior & 1 & 20 \\
\hline
\end{tabular}

\section{Treated group}

- Cases were grouped according to their histopathological diagnosis into Hodgkin and non-Hodgkin lymphoma.

- Ten cases diagnosed as Hodgkin while four cases diagnosed as non-Hodgkin lymphoma.

\section{A. Hodgkin lymphoma}

Nine cases presented by discrete and partially amalgamated lymph nodes affecting different groups of lymph nodes; while one case presented by mediastinal mass pathologically proved to be thymic hyperplasia (ADC value $=2.6)($ Fig. 3).
Six cases presented by restricted diffusion lymph nodes, 2 cases presented by facilitated diffusion lymph nodes and one case presented by restricted and facilitated diffusion lymph nodes (Fig. 4). The distribution of lymph nodes in treated Hodgkin lymphoma is demonstrated in Table 4.

ADC range in treated Hodgkin lymphoma cases presented with lymph node presentation was 1.04 to 3.6 with ADC average 1.573) (Fig. 4).

\section{B. Non-Hodgkin lymphoma}

Two cases presented by lymph nodes affection; while two cases presented by mediastinal mass (Fig. 5). One case presented by anterior mediastinal mass with ADC value 1.29. Another case showed anterior mediastinal mass with MRI findings and ADC value (2.72) suggestive of thymic hyperplasia (Table 5).

ADC range in treated non-Hodgkin lymphoma cases presented with lymph node presentation was 0.72 to 2.7 with ADC average 1.094) (Fig. 5).

\section{Statistics \\ Relations of ADC difference in Hodgkin and Non Hodgkin lymphoma}

There is statistic significant difference between ADC values in lymph nodes $(p=0.003)$ and mediastinal mass
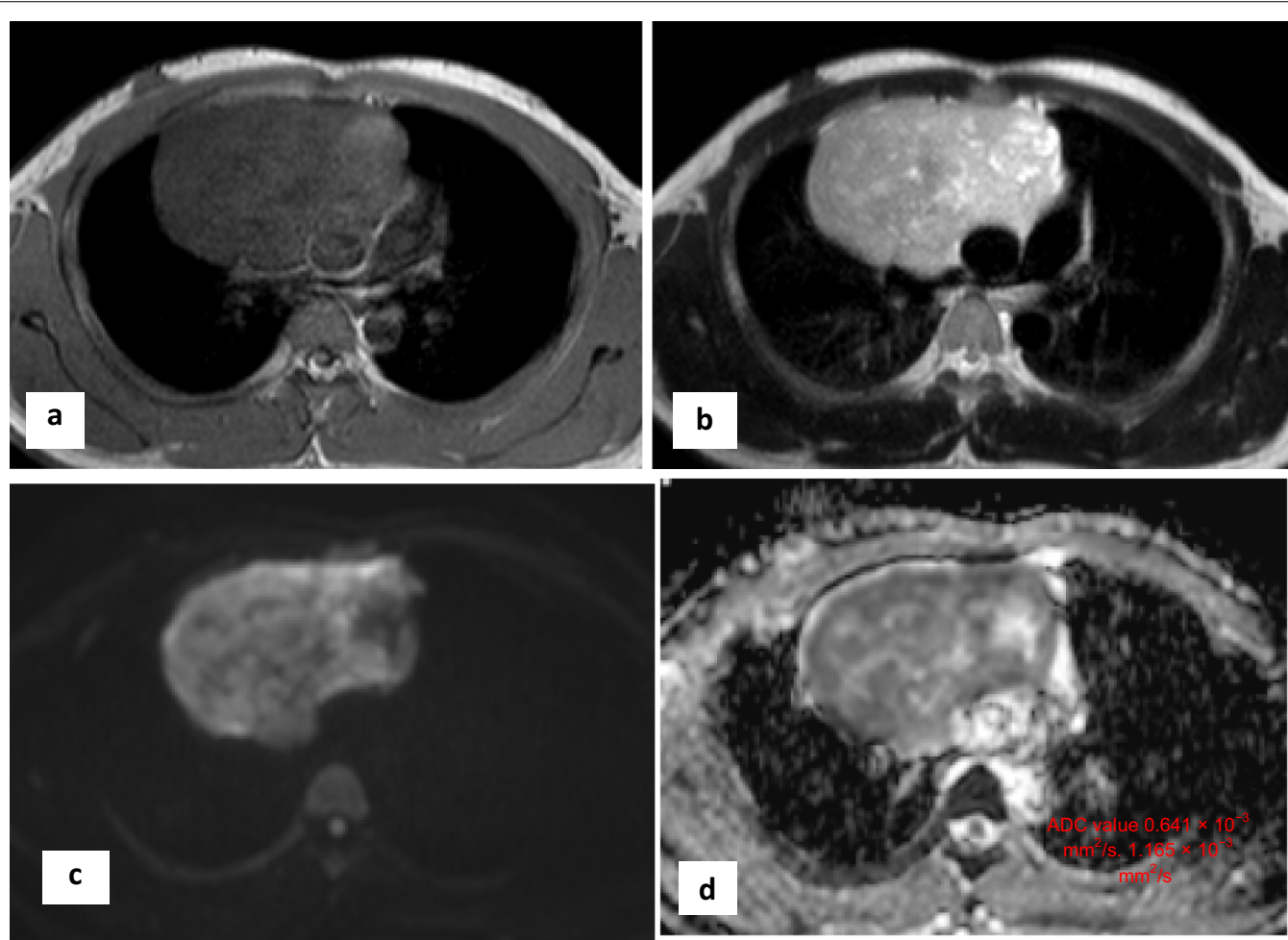

Fig. 2 A 20 year old female patient diagnosed as Non-Hodgkin lymphoma. MRI was done before onset of therapy. a-d: a Axial T1 WI, b axial T2 WI, c axial DWI and $\mathbf{d}$ ADC map show anterior mediastinal mass isointense on T1 WI, heterogeneous hyperintense on T2 WI, hyperintense on DWI and hypointense on ADC map denoting restricted diffusion with $A D C$ mean value $0.641 \times 10^{-3} \mathrm{~mm}^{2} / \mathrm{s}$ 

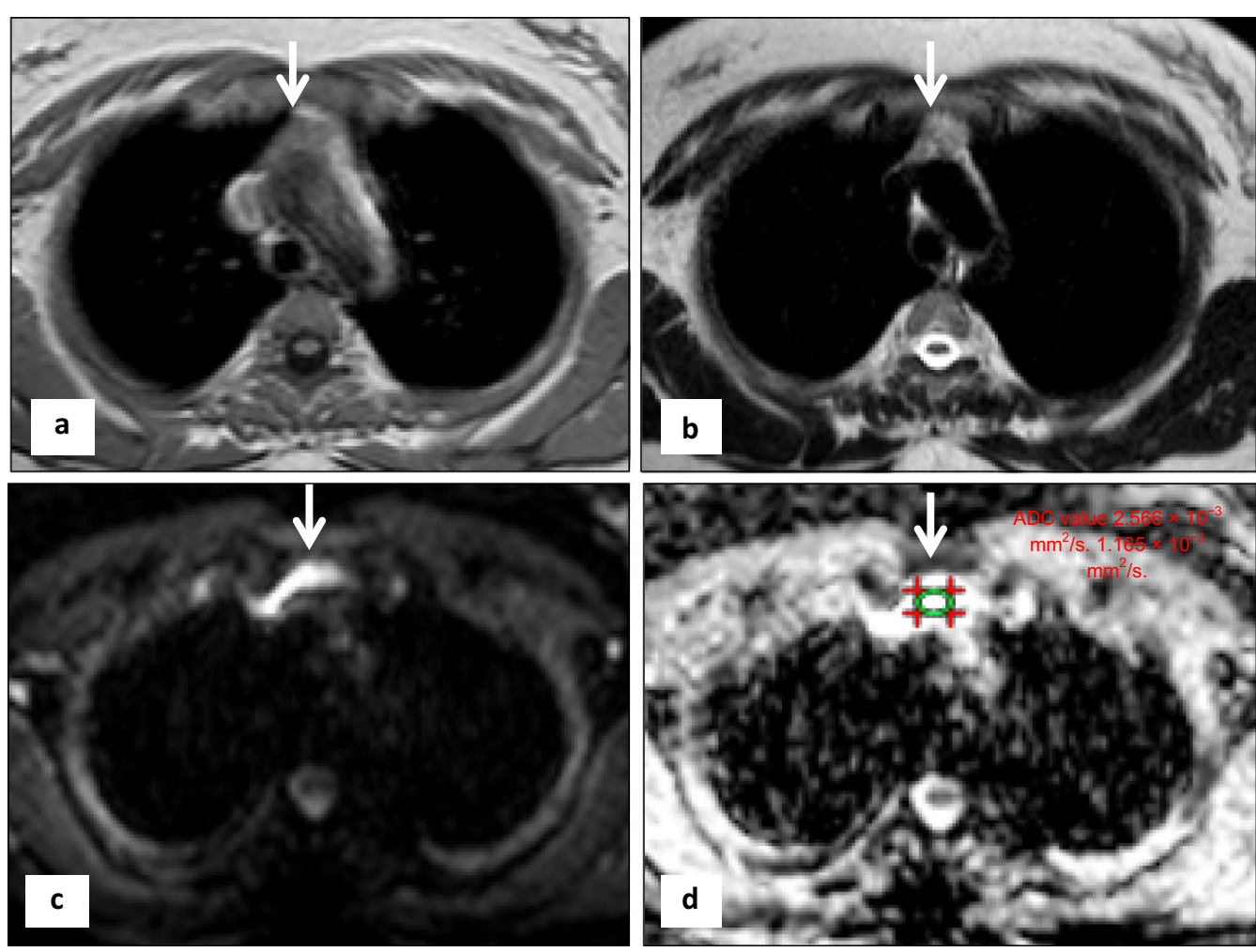

Fig. 3 A 30-year-old female diagnosed as Hodgkin lymphoma. MRI was done after starting receiving her chemotherapy sessions. a-d: a Axial T1 WI, $\mathbf{b}$ axial T2 WI, c axial DWI and $\mathbf{d}$ ADC map show anterior mediastinal mass lesion eliciting isointense signal in T1 and T2 WI, hyperintense signal in both DWI and ADC map with ADC value $2.566 \times 10^{-3} \mathrm{~mm}^{2} / \mathrm{s}$. The mass pathologically proved to be thymic hyperplasia

$(p=0.046)$ in Hodgkin and non-Hodgkin lymphomas (Table 6).

ADC cut off value to differentiate Hodgkin from nonHodgkin lymphoma in lymph nodes is 0.72 . Lymph node ADC value $\leq 0.72$ is diagnostic for non-Hodgkin lymphoma while $>0.72$ is diagnostic for Hodgkin lymphoma.

ADC cut off value to differentiate Hodgkin from nonHodgkin lymphoma in mediastinal mass is 0.38 . Mediastinal mass $A D C$ value $\leq 0.38$ is diagnostic for Hodgkin lymphoma while $>0.38$ is diagnostic for non-Hodgkin lymphoma.

\section{Relations of $A D C$ difference in treated and untreated lymphoma}

There is statistic significant difference between ADC values of mediastinal mass before and after chemotherapy $(p=0.002)$.

There is no statistical significant difference in results of chemotherapy in different lymph nodes groups (Fig. 6).

\section{Discussion}

Lymphoma represents one of the most common tumors in mediastinum approximately $10-15 \%$ of all masses $[18$, 19].
DWI is functional MR imaging technique with no need for special preparation, contrast injection or radiation exposure and allows the analysis of tissue characteristics based on the diffusivity of water molecules within tissues [20].

With the recent technology and advances in MR systems, diffusion weighted MRI (DWI MRI) of the chest is emerging as a promising fast technique that minimizes the effect of physiological motion artifacts that arise from respiration and cardiac movement [21].

The main purpose of this cross-sectional study was to assess the implementation of diffusion MRI in the algorithm of diagnosis of mediastinal lymphoma and assessment of post therapeutic response.

This study involved reviewing the DWI MRI finding results of 32 patients that were referred from the clinical oncology department for MRI assessment; all the patients were pathologically proved cases of lymphoma. Patients were grouped according to their histopathology and treatment status.

Out of 32 patients, 22 patients were diagnosed as Hodgkin lymphoma $(68.75 \%)$ and 10 patients were diagnosed as non-Hodgkin lymphoma (31.25\%). 

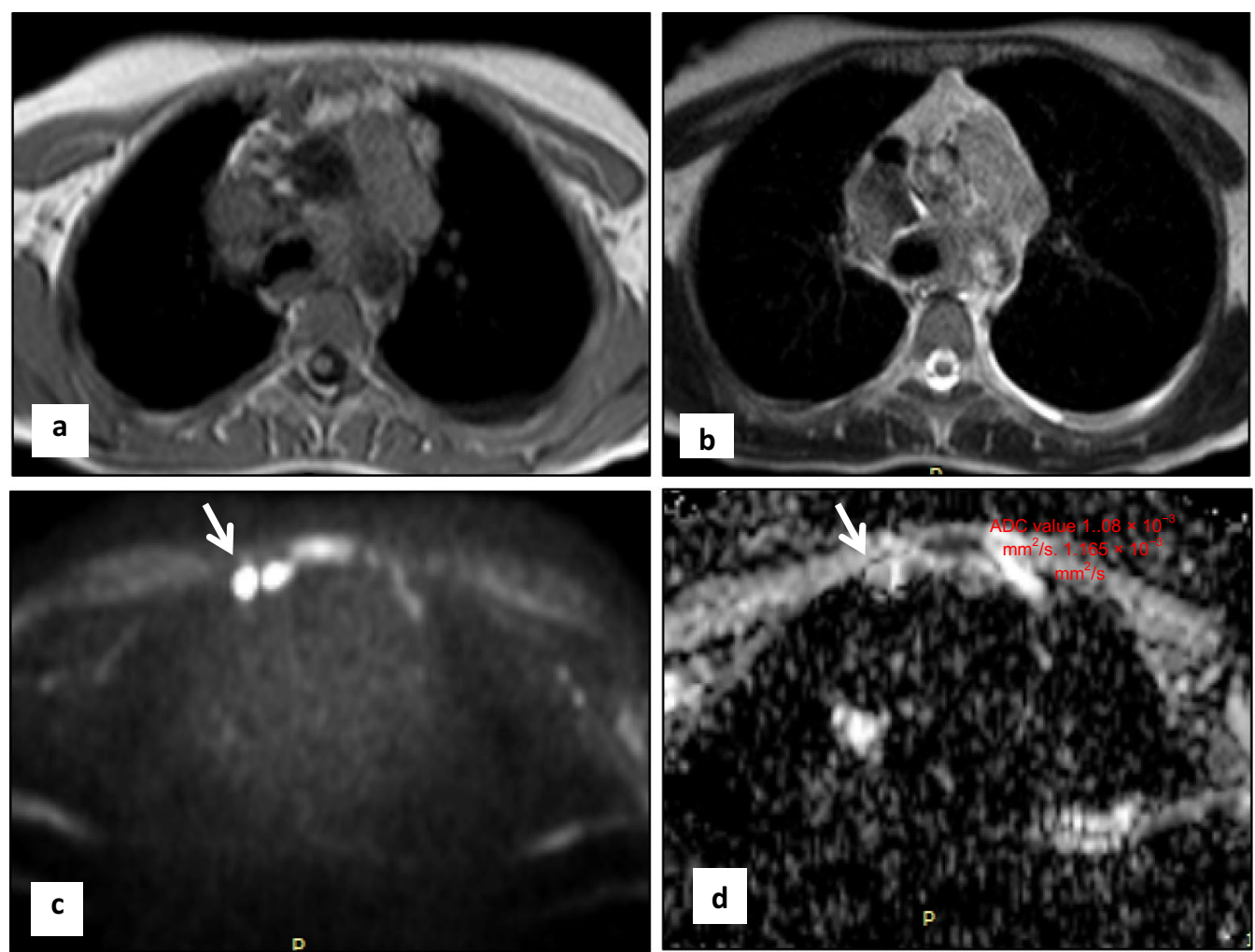

Fig. 4 A 23-year-old female diagnosed as Hodgkin lymphoma. MRI was done after starting receiving her chemotherapy sessions. a-d: a Axial T1 WI, $\mathbf{b}$ axial T2 WI, c axial DWI and $\mathbf{d}$ ADC map show enlarged prevascular, paratracheal and internal mammary lymph nodes, which are isointense on T1 and T2 WI. In DWI, only the internal mammary lymph nodes showed restricted diffusion in the form of high signal in DWI and low signal in ADC map "arrow" with ADC value $1.08 \times 10^{-3} \mathrm{~mm}^{2} / \mathrm{s}$, whereas the rest of mediastinal lymph nodes showed no evidence of diffusion restriction denoting good response to therapy

Table 4 Summary of lymph nodes distribution in treated Hodgkin lymphoma

\begin{tabular}{lll}
\hline Group of lymph node & Number of cases & Percentage \\
\hline Paratracheal & 1 & 6 \\
Pre vascular & 3 & 19 \\
Internal mammary & 1 & 6 \\
Hilar & 3 & 19 \\
Subcarinal & 2 & 13 \\
Paraesophageal & 2 & 13 \\
Cardiophernic & 4 & 25 \\
\hline
\end{tabular}

Out of 32 patients, MRI was done to 18 patients before starting treatment; 12 patients were diagnosed as Hodgkin lymphoma (66.66\%), and six patients were diagnosed as non-Hodgkin lymphoma (33.33\%). ADC range was 0.476 to 1.4 .

There is statistic significant difference between ADC values in LN $(p=0.003)$ and mediastinal masses $(p=0.046)$ in Hodgkin and non-Hodgkin lymphomas.
In agreement with Broncano et al. [22] and Çakmak et al. [23] who stated that, MRI DWI can be used as a reliable non-invasive technique that can differentiate between benign and malignant tumors including those of the mediastinum. This also consistent with Sabri et al. [21] and Abou Youssef et al. [24] who stated that the ADC values of malignant mediastinal lesions are significantly lower than those of benign lesions and determined cut-off ADC values to differentiate the two.

ADC range in non-treated Hodgkin lymphoma cases presented with lymph node presentation was 0.83 to 1.4 with ADC average 1.1072, while ADC range in cases presented with masses was 0.774 to 1.1 with average 0.939 . The ADC range in non-treated non-Hodgkin lymphoma cases presented with lymph node presentation was 0.476 to 0.548 with $\mathrm{ADC}$ average 0.512 , while $\mathrm{ADC}$ range in cases presented with masses was 0.507 to 0.668 with average 0.5946 . ADC cut off value to differentiate Hodgkin from non-Hodgkin lymphoma in lymph nodes was $0.72(p=0.003)$, while ADC cut off value to differentiate Hodgkin from non-Hodgkin lymphoma in mediastinal mass was $0.38(p=0.046)$. 

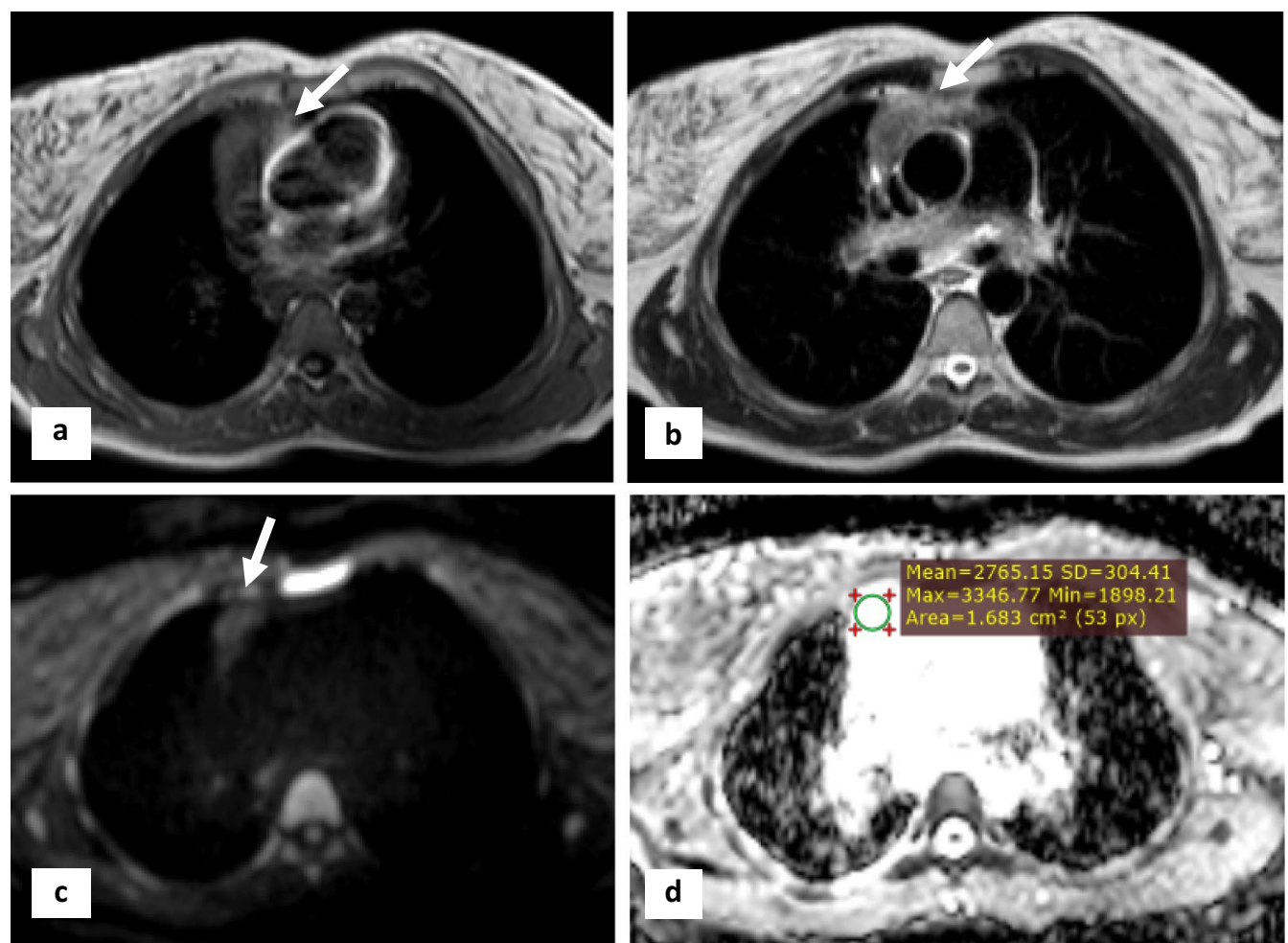

Fig. 5 A 36 year old female patient diagnosed as Non-Hodgkin lymphoma. MRI was done after receiving her chemotherapy sessions. a-d: a Axial $\mathrm{T} 1 \mathrm{WI}, \mathbf{b}$ axial $\mathrm{T} 2 \mathrm{WI}, \mathbf{c}$ axial DWI and $\mathbf{d} \mathrm{ADC}$ map show residual prevascular lymph node which is isointense on T1 and T2 WI, hyperintense on DWI and on ADC map with ADC value $2.8 \times 10^{-3} \mathrm{~mm}^{2} / \mathrm{s}$ denoting facilitated diffusion and good response to therapy

Table 5 Summary of lymph nodes distribution in treated nonHodgkin lymphoma

\begin{tabular}{lll}
\hline Group of lymph node & Number of cases & Percentage \\
\hline Paratracheal & 2 & 40 \\
Pre vascular & 1 & 20 \\
Para aortic & 1 & 20 \\
Cardiophrenic & 1 & 20 \\
\hline
\end{tabular}

On other hand Razek et al. [25] and Sabri et al. [21], stated regarding the pathological subtypes of mediastinal lymphoma, that there were no statistically difference between the ADC average value of Hodgkin lymphoma and non-Hodgkin lymphoma in these studies but the ADC value of non-Hodgkin lymphoma was lower than Hodgkin lymphoma. These findings may be attributed to limited study on relatively small sample size.

In this study out of 18 patients who did not start treatment at time of study eleven (61.1\%) cases presented by mediastinal masses and seven $(38.9 \%)$ cases present by lymph nodes. On searching the literature, no attempts to differentiate between lymphoma presentation as masses or discrete lymph nodes, in particular were found.

All of the eleven cases with mediastinal masses had the epicenter of the mass in the anterior mediastinum; which agrees with Shahrazad et al. [26] who stated that mediastinal lymphoma usually occur in the anterior mediastinum.

In the non-treated group of Hodgkin cases presented by discrete and partially amalgamated lymph nodes, the distribution of lymph nodes was as follows: Enlarged para-tracheal lymph node were in five cases (19\%), enlarged prevascular lymph nodes were in four cases (15\%), enlarged para-aortic lymph nodes were in two cases (8\%), enlarged sub-aortic lymph nodes were in three cases $(11.5 \%)$, enlarged hilar lymph nodes were three cases (11.5\%), enlarged subcarinal lymph nodes were in two cases(8\%). Enlarged para-esophageal lymph nodes were in four cases (15\%), enlarged cardio-phrenic was in one case (4\%).enlarged supra clavicular lymph nodes was in one case (4\%) and enlarged internal mammary lymph nodes was in one case (4\%).

That is consistent with Mehrian et al. [27] study on patients with Hodgkin's disease, enlarged lymph nodes were distributed mainly in the following zones: 4 (lower 
Table 6 Mann-Whitney Test

\begin{tabular}{|c|c|c|c|c|c|}
\hline & \multicolumn{5}{|l|}{ Ranks } \\
\hline & Pathology & $\underline{\mathrm{N}}$ & Mean rank & Sum of ranks & $p$ value \\
\hline \multirow[t]{3}{*}{ ADC of paratracheal } & Hodgkin & 5 & 4.00 & 20.00 & \\
\hline & Non Hodgkin & 2 & 4.00 & 8.00 & 1.000 \\
\hline & Total & 7 & & & \\
\hline \multirow[t]{3}{*}{ ADC prevascular } & Hodgkin & 6 & 4.50 & 27.00 & 0.134 \\
\hline & Non Hodgkin & 1 & 1.00 & 1.00 & \\
\hline & Total & 7 & & & \\
\hline \multirow[t]{3}{*}{ ADC hilar } & Hodgkin & 6 & 3.50 & 21.00 & \\
\hline & Non Hodgkin & $0^{\mathrm{a}}$ & .00 & .00 & - \\
\hline & Total & 6 & & & \\
\hline \multirow[t]{3}{*}{ ADC value average } & Hodgkin & 21 & 19.33 & 406.00 & \\
\hline & Non Hodgkin & 10 & 9.00 & 90.00 & 0.003 \\
\hline & Total & 31 & & & \\
\hline \multirow[t]{3}{*}{ ADC mediastinal Mass average } & Hodgkin & 22 & 14.48 & 318.50 & \\
\hline & Non Hodgkin & 10 & 20.95 & 209.50 & 0.046 \\
\hline & Total & 32 & & & \\
\hline \multirow[t]{3}{*}{ ADC anterior mediastinal mass } & Hodgkin & 7 & 5.14 & 36.00 & \\
\hline & Non Hodgkin & 5 & 8.40 & 42.00 & 0.123 \\
\hline & Total & 12 & & & \\
\hline
\end{tabular}

${ }^{\text {a }}$ Mann-Whitney Test cannot be performed on empty groups

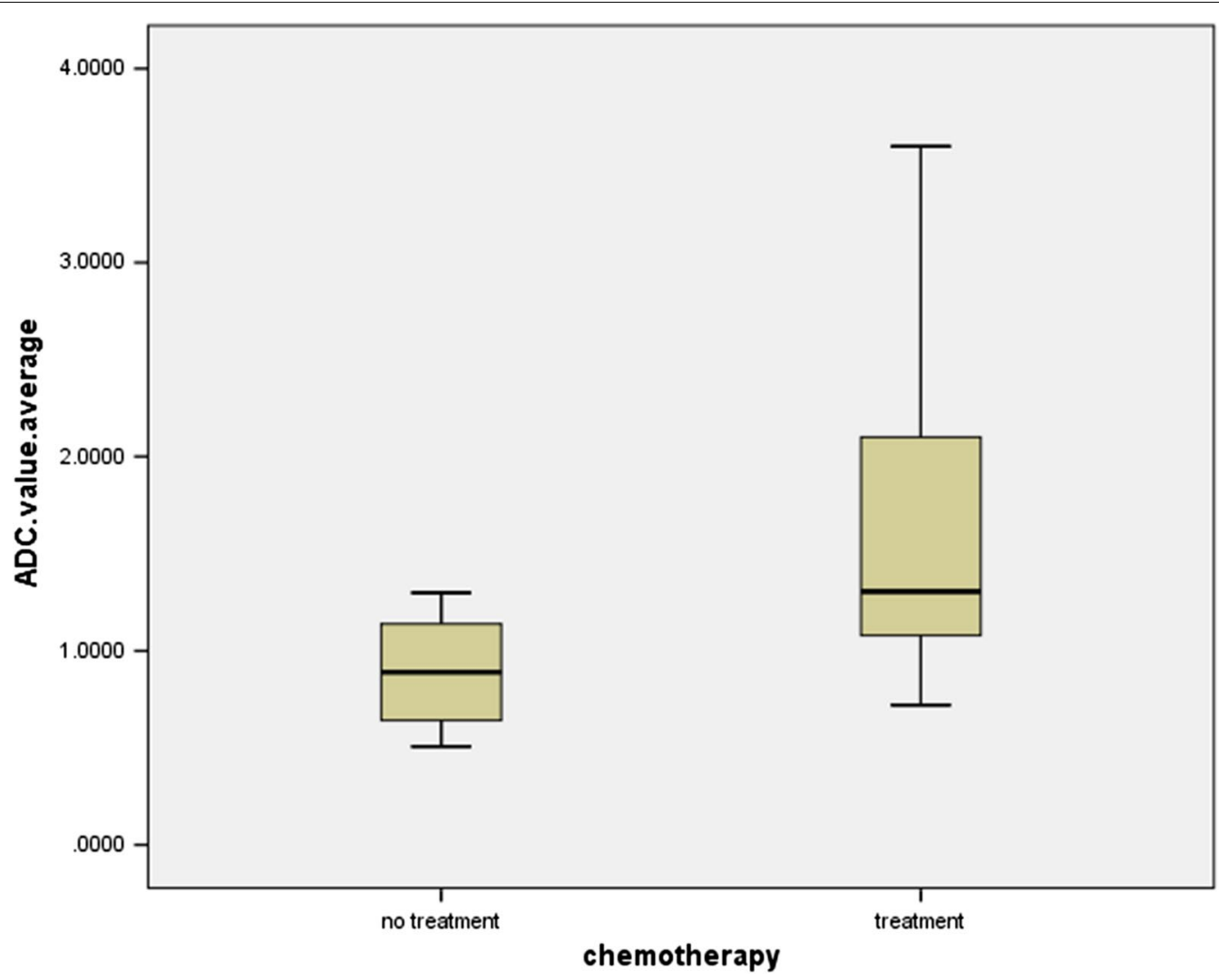

Fig. 6 Bar-and-Whisker plot: ADC mean values of NHL and HD 
paratracheal; 89.2\%), 3 (prevascular; 81.1\%), 7 (subcarinal; 73.0\%) and 2 (upper paratracheal; 70.2\%). Zone nine (pulmonary ligament) and peri-diaphragmatic zones were rarely affected in either Hodgkin's lymphoma.

Out of fourteen patients with MRI done after starting chemotherapy sessions; ten patients were diagnosed as Hodgkin lymphoma (71.428\%), and four patients were diagnosed as non-Hodgkin lymphoma (28.571\%). ADC range in treated Hodgkin lymphoma cases presented with residual lymph node presentation was 1.04 to 3.6 with ADC average 1.573. ADC range in treated non-Hodgkin lymphoma cases presented with lymph node presentation was 0.72 to 2.7 with ADC average 1.094, while ADC mean in one case presented with mass was 1.29.

In this study, there was statistically significant difference of ADC values in cases presented by masses before and after chemotherapy, while there was statistically insignificant difference of ADC values in case presented by lymph nodes before and after chemotherapy. These findings may be attributed to relatively small sample size.

This is agrees with Lin et al. [28], who stated that functional information provided by diffusion MRI are potentially helpful in assessment of response to treatment. Littooij et al. [29] stated that ADC measurements could be a valuable for the differentiation between viable and non-viable residual lesions.

De Paepe et al. [14] stated that DWI may be used as a response marker very early during treatment for lymphoma.

In two cases who had received chemotherapy, there was a residual anterior mediastinal mass lesion that was considered as residual lymphoma; however their ADC values were 2.6 and 2.7 , suggesting benign nature rather than lymphoma residual. The possibility of thymic hyperplasia was considered and was proved by histopathology.

In agreement with Zhen et al. [30] who stated that thymus hyperplasia following chemotherapy can occur in both children and adults, but occurs most often in children, adolescents and young adults. It can occur in various types of tumors including lymphoma.

One case $(7 \%)$ showed multiple residual lymph nodes; most of them showing facilitated diffusion denoting benign nature while only two showed restricted diffusion with ADC value reflecting malignancy. In agreement with Chowdhury et al. [31] and Spiekermann et al. [32] who stated that sarcoid like reactions have been described in association with many different types of malignancy.

In agreement with Sabri et al. [17] and Ibrahim et al. [33], who stated that DWI MRI can differentiate benign from malignant mediastinal masses and can differentiate lymphoma from sarcoidosis in the setting of mediastinal and hilar lymphadenopathy.
However as many studies, this study encompasses some limitations. First of them is the susceptibility artifacts despite the usage of phase array coil with cardiac gating and respiratory compensation techniques to improve image quality and speed. However, these artifacts were not severe enough to interfere with the diagnostic information gained by different MR sequences.

The second limitation is the relatively small number of cases, so we recommend further research on a larger population to confirm our results.

\section{Conclusions}

We concluded that DWI MRI is a promising functional technique in diagnosis of Hodgkin and non-Hodgkin lymphoma and assessment of response to treatment with significant statistic difference between ADC values of lymph nodes and mediastinal mass in Hodgkin and non-Hodgkin lymphomas.

\section{Abbreviations}

DWI: Diffusion weighted imaging; MRI: Magnetic resonance imaging; IASLC: International Association for the Study of Lung Cancer; ADC: Apparent Diffusion Coefficient; 18-FDG PET: 18 Fluoro-deoxy glucose positron-emission tomography; CT: Computed tomography; WI: Weighted imaging; $\mathrm{mm}^{2} / \mathrm{s}$ : Square millimeter per Seconds; STIR: Short T1 inversion recovery; TR: Echo time; TE: Repetition time; TSE: Turbo spin echo; FOV: Field of view; mm: Millimeter; mms: Millimeter second.

\section{Acknowledgements}

The authors would like to thank all the personnel contributed in this study.

\section{Authors' contributions}

Y.Y. shared in study conception and design, writing and correcting the manuscript and revision. H.Z. shared in study conception and design, acquisition of data, analysis and interpretation of data and drafting of manuscript. N.E. shared in collecting patients' data, processing MRI findings at MR work station and shared in writing the manuscript. M.K. shared in study conception and design, acquisition of data, analysis and interpretation of data and drafting of manuscript. All authors read and approved the final manuscript.

Funding

This study had no funding from any resource.

\section{Declarations}

\section{Ethics approval and consent to participate}

No individual data included in the study. The study was approved by the Research Ethics Committee of the Faculty of Medicine at Cairo University; reference number: Code: D-34-2019. All patients included in this study gave verbal and written informed consent to participate in this research.

\section{Consent for publication \\ Not applicable.}

\section{Availability of data and material}

The datasets used and/or analyzed during the study are available upon reasonable request.

\section{Competing interests}

The authors declare that they have no competing interests. 


\section{Author details}

${ }^{1}$ Department of Diagnostic and Interventional Radiology, Kasr Al Ainy, Faculty of Medicine, Cairo University, Giza City, Egypt. ${ }^{2}$ Department of Clinical Oncology, Kasr Al Ainy, Faculty of Medicine, Cairo University, Giza City, Egypt.

Received: 13 April 2021 Accepted: 23 August 2021

Published online: 31 August 2021

\section{References}

1. Cunningham J, lyengar S, Sharma B (2017) Evolution of lymphoma staging and response evaluation: current limitations and future directions. Nat Rev Clin Oncol 14(10):631-645

2. Rusch VW, Asamura H, Watanabe $\mathrm{H}$ et al (2009) The IASLC lung cancer staging project: a proposal for a new international lymph node map in the forthcoming seventh edition of the TNM classification for lung cancer. J Thoracic Oncol 4(5):568-577

3. El-Sherief AH, Lau CT, Obuchowski NA et al (2017) Cross-disciplinary analysis of lymph node classification in lung cancer on CT scanning. Chest 151(4):776-785

4. Park BJ, Kim TH, Shin S et al (2019) Recommended change in the $\mathrm{N}$ descriptor proposed by the International Association for the Study of Lung Cancer: a validation study. J Thorac Oncol 14(11):1962-1969

5. Balbo-Mussetto A, Cirillo S, Bruna R et al (2016) Whole-body MRI with diffusion-weighted imaging: a valuable alternative to contrast-enhanced CT for initial staging of aggressive lymphoma. Clin Radiol 71(3):271-279

6. Sigovan M, Akl P, Mesmann C, Tronc F, Si-Mohamed S, Douek P, Boussel $L$ (2018) Benign and malignant enlarged chest nodes staging by diffusion-weighted MRI: an alternative to mediastinoscopy. Br J Radiol 91(1082):20160919

7. Chavhan GB, AISabban Z, Babyn PS (2014) Diffusion-weighted imaging in pediatric body MR imaging: principles, technique, and emerging applications. Radiographics 34(3):E73-E88

8. Bajada CJ, Parker GJ, Lambon Ralph MA et al (2017) Diffusion MRI: from basic principles to clinical applications. Malta Med School Gazette 1(2):41-47

9. Carter BW, Wu CC, Khorashadi L et al (2014) Multimodality imaging of cardiothoracic lymphoma. Eur J Radiol 83(8):1470-1482

10 Priola AM, Galetto G, Priola SM (2014) Diagnostic and functional imaging of thymic and mediastinal involvement in lymphoproliferative. Clin Imaging 38(6):771-784

11. Takahashi K, Al-Janabi NJ (2010) Computed tomography and magnetic resonance imaging of mediastinal tumors. J Magn Reson Imaging 32(6):1325-1339

12. Razek AA, Elkammary S, Elmorsy AS et al (2011) Characterization of mediastinal lymphadenopathy with diffusion-weighted imaging. Magn Reson Imaging 29(2):167-172

13. Ranganath $\mathrm{SH}$, Lee EY, Restrepo R et al (2012) Mediastinal masses in children. Am J Roentgenol 198(3):W197-W216

14. De Paepe K, Bevernage C, De Keyzer F et al (2013) Whole-body diffusionweighted magnetic resonance imaging at 3 Tesla for early assessment of treatment response in non-Hodgkin lymphoma: a pilot study. Cancer Imaging 13(1):53

15. Gümüştaş S, Inan N, Sarisoy HT et al (2011) Malignant versus benign mediastinal lesions: quantitative assessment with diffusion weighted MR imaging. Eur Radiol 21(11):2255-2260

16. Yabuuchi H, Matsuo Y, Abe K, Baba S et al (2015) Anterior mediastinal solid tumours in adults: characterisation using dynamic contrast-enhanced MRI, diffusion-weighted MRI, and FDG-PET/CT. Clin Radiol 70(11):1289-1298

17. Sabri YY, Kolta MFF, Khairy MA (2017) MR diffusion imaging in mediastinal masses the differentiation between benign and malignant lesions. Egypt J Radiol Nucl Med 48(3):569-580

18. Akshatha RA, Seshadri S, Teerthanath S et al (2014) A study of clinical characteristicsof mediastinal mass. J Clin Diagn Res: JCDR 8(2):77

19 Chira RI, Chira A, Mircea PA et al (2017) Mediastinal masses-transthoracic ultrasonography aspects. Medicine 96(49):e9082

20. Hagtvedt T, Seierstad T, Lunet KV et al (2015) Diffusion-weighted MRI compared to FDG PET/CT for assessment of early treatment response in lymphoma. Acta Radiol 56(2):152-158

21. Sabri YY, Nossair EZB, Assal HH et al (2020) Role of diffusion weighted MR-imaging in the evaluation of malignant mediastinal lesions. Egypt J Radiol Nucl Med 51(1):32

22. Broncano J, Alvarado-Benavides AM, Bhalla S et al (2019) Role of advanced magnetic resonance imaging in the assessment of malignancies of the mediastinum. World J Radiol 11(3):27

23. Çakmak V, Ufuk F, Karabulut N (2017) Diffusion-weighted MRI of pulmonary lesions: comparison of apparent diffusion coefficient and lesion-tospinal cord signal intensity ratio in lesion characterization. J Magn Reson Imaging 45(3):845-854

24 Abou Youssef HA, Elzorkany MA, Hussein SAM et al (2019) Evaluation of mediastinal lymphadenopathy by diffusion weighted MRl; correlation with histopathological results. Adv Resp Med 87(3):175-183

25. Razek AA, Gaballa G, Elashry R et al (2015) Diffusion-weighted MR imaging of mediastinal lymphadenopathy in children. Jpn J Radiol 33(8):449-454

26. Shahrzad M, Le TSM, Silva M et al (2014) Anterior mediastinal masses. Am J Roentgenol 203(2):W128-W138

27. Mehrian P, Ebrahimzadeh SA (2013) Differentiation between sarcoidosis and Hodgkin's lymphoma based on mediastinal lymph node involvement pattern: evaluation using spiral CT scan. Pol J Radiol 78(3):15

28. Lin C, Lucianib A, Itti E et al (2012) Whole-body diffusion magnetic resonance imaging in the assessment of lymphoma. Cancer Imaging 12(2):403

29. Littooij AS, Kwee TC, de Keizer B et al (2015) Whole-body MRI-DWI for assessment of residual disease after completion of therapy in lymphoma: a prospective multicenter study. J Magn Reson Imaging 42(6):1646-1655

30. Zhen Z, Sun X, Xia Y et al (2010) Clinical analysis of thymic regrowth following chemotherapy in children and adolescents with malignant lymphoma. Jpn J Clin Oncol 40(12):1128-1134

31. Chowdhury FU, Sheerin F, Bradley KM et al (2009) Sarcoid-like reaction to malignancy on whole-body integrated 18F-FDG PET/CT: prevalence and disease pattern. Clin Radiol 64(7):675-681

32. Spiekermann C, Kuhlencord M, Huss S et al (2017) Coexistence of sarcoidosis and metastatic lesions: a diagnostic and therapeutic dilemma. Oncol Lett 14(6):7643-7652

33. Ibrahim TE, Elia RZ, Hussien RS et al (2020) MR diffusion imaging in mediastinal masses the differentiation between benign and malignant lesions. QJM: Int J Med 113(Supplement_1):pp.hcaa068-008

\section{Publisher's Note}

Springer Nature remains neutral with regard to jurisdictional claims in published maps and institutional affiliations. 